\title{
COVID-19 and male reproductive function: a prospective, longitudinal cohort study
}

\author{
Behzad Hajizadeh Maleki ${ }^{1}$ and Bakhtyar Tartibian² \\ ${ }^{1}$ Department of Exercise Physiology and Sports Therapy, Kugelberg 62, Justus-Liebig-University, Giessen, Germany \\ and ${ }^{2}$ Department of Sports Injuries, Faculty of Physical Education and Sports Sciences, Allameh Tabataba'i \\ University, Tehran, Iran
}

Correspondence should be addressed to B Hajizadeh Maleki; Email: hajizadeh.sci.phys@gmail.com

\begin{abstract}
The existing evidence suggests that the human reproductive system may be potentially vulnerable to COVID-19 infection. However, little is known about the virus-host interaction of COVID-19 in sperm cells. We are the first to address the connection between changes in multiple seminal biomarkers and reproductive function in male patients recovering from COVID-19. In a prospective longitudinal cohort study, seminal ACE2 activity, markers of inflammation and oxidative stress, apoptotic variables, and semen quality parameters were evaluated at 10-day intervals for a maximum follow-up time of $\mathbf{6 0}$ days among male patients with laboratoryconfirmed COVID-19 $(n=84)$ and healthy controls $(C O N ; n=105)$. At the baseline and the subsequent follow-ups, the COVID-19 group revealed significantly higher levels of seminal plasma ACE2 enzymatic activity, IL-1 $\beta$, IL-6, IL-8, IL-10, TGF- $\beta$, TNF- $\alpha$, IFN- $\alpha$, IFN- $\gamma$, ROS, caspase-8, caspase-9, and caspase-3 activity as well as lower levels of SOD activity than those in the CON group $(P<$ 0.05). These perturbations tended to persist over time and were correlated with significant impairments in semen volume, progressive motility, sperm morphology, sperm concentration, and the number of spermatozoa. We provide the direct experimental evidence that the male reproductive system could be targeted and damaged by the COVID-19 infection. These findings go beyond our current understanding of the disease, suggesting that the reproductive function of the patients recovering from the disease should be precisely followed and evaluated to detect and avoid more serious reproductive problems in the future, as they may develop a transient state of male subfertility like those with oligoasthenoteratozoospermia.

Reproduction (2021) 161 319-331
\end{abstract}

\section{Introduction}

Since the first report in Wuhan, Hubei Province of China, in late December 2019, the novel SARS-CoV-2 (severe acute respiratory syndrome coronavirus 2) has spread worldwide exponentially, leading to the coronavirus disease (COVID-19) being confirmed a global pandemic outbreak by the World Health Organization (WHO) on the March 11, 2020. As of December 25, 2020, over 79 million cases of COVID-19 have been confirmed, and approximately $1,740,423$ official deaths have been declared globally. The virus that causes COVID-19 leads to acute respiratory distress syndrome with severe respiratory symptoms and relatively high fatality risk (Chan et al. 2020, Chen et al. 2020, Huang et al. 2020). It is predominantly transmitted via close contact with an infected patient and with infected large respiratory droplets propagated when an infected person speaks, exhales, sneezes, or coughs (Chan et al. 2020, Liu et al. 2020b). Recent structural and functional evidence reported that due to having a strong binding affinity to the human cell receptor, angiotensin-converting enzyme 2 (ACE2), COVID-19 employs this receptor, in synergy with the host's type II transmembrane serine protease (TMPRSS2) (Mollica et al. 2020), for entry into target host cells (Hoffmann et al. 2020, Zhao et al. 2020). Accordingly, the cells expressing these receptors may function as the target cells and consequently might let COVID-19 entry, proliferation, propagation, and pathogenesis (Chai et al. 2020, Mollica et al. 2020, Zhang et al. 2020, Zhou et al. 2020, Zou et al. 2020). It has been declared that ACE2 and TMPRSS2 are highly expressed not only in the lungs, kidney, intestine, and heart tissues (Gkogkou et al. 2020, Zhou et al. 2020) but in the spermatogonia, Leydig, and Sertoli cells in the human testes (Fan et al. 2020), as well as in other male reproductive organs like the prostate gland (Maya \& Carvajal 2020), the seminal vesicles, and the bulbourethral glands (Zupin et al. 2020), that contribute seminal fluid to the semen, suggesting that COVID-19 could infect the male reproductive organs and risk male reproduction (Fan et al. 2020). In this regard, the impaired spermatogenesis (Li et al. 2020) and declined sperm quality parameters (Ruan et al. 2020) have already been detected in COVID-19 patients. However, the association and the infection-induced-longitudinal changes in the male reproductive function in COVID-19 
patients are not clear. Also, the exact mechanisms behind these observations remain an under-researched issue.

To address this query, therefore, we examined the temporal changes in seminal ACE2 enzymatic activity, pro- and anti-inflammatory cytokines, oxidative and antioxidative parameters, apoptotic variables, and semen quality parameters in reproductive-aged Iranian male patients recovering from COVID-19 and agematched healthy controls, measured at 10-day intervals for a maximum follow-up time of 60 days, and defined their associations with male reproductive function.

\section{Methods \\ Participants and study design}

In a prospective longitudinal cohort study, male patients (aged 20-40 years) with laboratory-confirmed COVID-19, hospitalized in Masih Daneshvari Hospital, Tehran, Tehran province, Iran, between March 20, 2020, and April 20, 2020, were identified. Laboratory confirmation for COVID-19 was based on the WHO's guidelines (WHO 2020). It was defined as a positive result for SARS-CoV-2 in the qRT-PCR assay of nasal and pharyngeal swab specimens (Huang et al. 2020). The diagnosis and severity of COVID-19 were determined based on the 7th edition of the New Coronavirus Pneumonia Prevention and Control Program printed through China's National Health Commission.

Before initiating the study, patients were first identified and provided with a study information booklet through nurse managers in the units as a third-party. Patients with proven fertility, based on physical examination and history, who were willing to participate in the study, gave their written informed consent, exchanged the contact information with the research team, and were asked to contact them before being discharged from the hospital. Accordingly, all respective patients $(n=116)$ were approached by a trained research assistant within $24 \mathrm{~h}$ after being discharged from the hospital. Patients received requisite information in the written and spoken form, and their personal data and physical characteristics were collected by investigators (Table 1). Besides, recruited through a community advertisement, following an extensive screening, a total of 108 sedentary, healthy men (aged 20-40 years) with proven fertility were included as a control (CON) group after signing a written informed consent (Table 1). Thirty-five participants (COVID-19, $n=32$; CON, $n$ $=3$ ) could not complete the study protocol and were excluded from the study. Thus, 189 participants (COVID-19, $n=84$; CON, $n=105)$ were finalized for the investigation. All the men had normal outcomes on fertility evaluation as proven by a Urology expert, and preexisting conditions and infertile factors were excluded. This study was approved by the Ethics Committee of Allameh Tabataba'i University, Tehran, Iran, and was conducted in accordance with the Declaration of Helsinki.

\section{Samplings}

All the participants were clearly instructed on collecting their semen samples into a sterile container by masturbation. Following 3-4 days of sexual abstinence, participants in the
Table 1 Demographic and clinical characteristics of patients and participants at baseline. The values are presented as the mean \pm S.D. or as $\mathrm{n}(\%)$.

\begin{tabular}{|c|c|c|}
\hline Characteristics & $\begin{array}{l}\text { COVID-19 } \\
(n=84)\end{array}$ & $\begin{array}{l}\text { CON } \\
(n=105)\end{array}$ \\
\hline Age (years) & $34.7 \pm 6.3$ & $33.9 \pm 7.5$ \\
\hline \multicolumn{3}{|l|}{ Age category (years), $n(\%)$} \\
\hline $20-25$ & $8(9.6 \%)$ & $14(13.3 \%)$ \\
\hline $26-30$ & $18(21.4 \%)$ & $22(21.0 \%)$ \\
\hline $31-35$ & $28(33.3 \%)$ & $35(33.3 \%)$ \\
\hline $36-40$ & $30(35.7 \%)$ & $34(32.4 \%)$ \\
\hline \multicolumn{3}{|l|}{ Severity, $n(\%)$} \\
\hline Mild & $1(1.2 \%)$ & - \\
\hline Moderate & $23(27.4 \%)$ & - \\
\hline Severe & $27(32.1 \%)$ & - \\
\hline Critical & $33(39.3 \%)$ & - \\
\hline \multicolumn{3}{|l|}{ Symptoms, $n(\%)$} \\
\hline Fever & $83(98.8 \%)$ & - \\
\hline Cough & $62(73.8 \%)$ & - \\
\hline Sore throat & $47(55.9 \%)$ & - \\
\hline Myalgia & $69(82.1 \%)$ & - \\
\hline Dyspnoea & $74(88.1 \%)$ & - \\
\hline Chest pain & $66(78.6 \%)$ & - \\
\hline Diarrhoea & $57(67.9 \%)$ & - \\
\hline $\begin{array}{l}\text { Time between diagnosis } \\
\text { to the first sampling (day) }\end{array}$ & $13.2 \pm 4.9$ & - \\
\hline Weight $(\mathrm{kg})$ & $70.7 \pm 12.3^{*}$ & $83.8 \pm 9.1^{*}$ \\
\hline Fat $(\%)$ & $13.1 \pm 5.8^{*}$ & $22.9 \pm 6.9^{*}$ \\
\hline $\mathrm{BMI}\left(\mathrm{kg} / \mathrm{m}^{2}\right)$ & $23.4 \pm 6.2^{*}$ & $27.4 \pm 6.5^{*}$ \\
\hline \multicolumn{3}{|l|}{ BMI category } \\
\hline$<25 \mathrm{~kg} / \mathrm{m}^{2}$ & $60(71.4 \%)$ & $55(52.4 \%)$ \\
\hline $25-29.99 \mathrm{~kg} / \mathrm{m}^{2}$ & $22(26.2 \%)$ & $48(45.7 \%)$ \\
\hline$\geq 30 \mathrm{~kg} / \mathrm{m}^{2}$ & $2(2.4 \%)$ & $2(1.9 \%)$ \\
\hline Waist circumference $(\mathrm{cm})$ & $98.6 \pm 7.4^{*}$ & $\begin{array}{l}124.7 \pm \\
22.3^{*}\end{array}$ \\
\hline SARS-CoV-2 semen result & Negative & - \\
\hline \multicolumn{3}{|l|}{ Marital status, $n(\%)$} \\
\hline Married/living with partner & $66(78.6 \%)$ & $81(77.1 \%)$ \\
\hline Divorced/separated & $8(9.5 \%)$ & $12(11.4 \%)$ \\
\hline Widowed & $2(2.4 \%)$ & $5(4.8 \%)$ \\
\hline Never married & $8(9.5 \%)$ & $7(6.7 \%)$ \\
\hline Number of children & $2.4 \pm 1.1$ & $2.2 \pm 0.9$ \\
\hline Presence of urogenital disease & No & No \\
\hline Corticosteroid therapy & $37(44.0 \%)$ & - \\
\hline Antiviral therapy & $58(69.0 \%)$ & - \\
\hline
\end{tabular}

${ }^{*} P<0.05$, significant difference between the groups. $\mathrm{CON}$, control group.

CON group reported to the lab on the respective sampling days to provide semen samples at the site. In the COVID19 group, the patients who could not attend the laboratory due to their health condition and whose distance from the laboratory was close $(n=9 ; 10.7 \%)$, samples were collected at home and delivered to the laboratory through a portable incubator (Minitüb GmbH, Tiefenbach - Germany) within 30 min of collection by a researcher wearing suitable personal protective equipment. The remaining patients $(n=75$; $89.3 \%$ ) were provided semen samples on-site. The duration of sexual abstinence before taking the semen sample was not standardized for the COVID-19 group. Semen samples were taken at baseline ( $24 \mathrm{~h}$ after hospital discharge) and follow-ups at 10, 20, 30, 40, 50, and 60 days after baseline. Using an identical protocol, SARS-CoV-2 detection in semen samples 
was performed (Huang et al. 2020). The time between the confirmatory diagnosis of COVID-19 and first semen collection was $13.2 \pm 4.9$ days.

\section{Analysis and measurements}

Following at least $30 \mathrm{~min}$ of liquefaction, semen analysis was performed in accordance with the WHO guidelines for the examination of human semen to assess semen quality parameters (i.e. semen volume, sperm motility, sperm morphology, sperm concentration, and the number of spermatozoa) (World Health Organization 2010). Briefly, after liquefaction, semen volume was assessed with graduation of $0.1 \mathrm{~mL}$ by a wide-bore graduated pipette. Sperm concentration and motility were evaluated at a magnification of $\times 200$ or 400 under a phase-contrast microscope (OLYMPUS BX43). For the sperm concentration, when necessary, standard dilutions were applied, and counting was done following 10-15 min of sedimentation by hemocytometers with improved Neubauer ruling. For sperm motility, a wet preparation was made in duplicate with a drop of $10 \mu \mathrm{L}$ semen sample and a $22 \mathrm{~mm} \times 22 \mathrm{~mm}$ coverslip to give a depth of $20 \mu \mathrm{m}$, and at least 200 spermatozoa were evaluated each time. Tygerberg strict criteria were used to assess sperm morphology following staining the slides with a Diff-Quik staining kit (Dade Behring AG, Switzerland). The measurements were done under a microscope with an oil immersion of $\times 100$ objectives (OLYMPUS BX43). All analyses were performed by one experienced technician who was blinded to the study. No differences in liquefaction times were observed between the two groups, and semen samples liquefied within 20 to $30 \mathrm{~min}$ of ejaculation. After $10 \mathrm{~min}$ of centrifugation at $10,000 \mathrm{~g}$, the supernatant seminal plasma was preserved at $-80^{\circ} \mathrm{C}$ until the examination (Hajizadeh Maleki \& Tartibian 2020). The seminal plasma then was used to assess ACE2 enzymatic activity, interleukin (IL)-1 $\beta$, IL-6, IL-8, IL-10, tumor necrosis factor-alpha (TNF- $\alpha$ ), interferon-alpha (IFN- $\alpha$ ), IFN- $\gamma$, transforming growth factor- $\beta$ (TGF- $\beta$ ), reactive oxygen species (ROS), superoxide dismutase (SOD), caspase-8, caspase-9, and caspase-3 activity.

\section{ACE2 activity assay}

As described previously (Douglas et al. 2004), an ACE2 quenched fluorescent substrate (QFS, (7-methoxycoumarin4-yl)acetyl-Ala-Pro-Lys (2,4-dinitrophenyl)) was used to detect the catalytic activity of ACE2. Using the specific inhibitor MLN-4760 at $100 \mathrm{nmol} / \mathrm{L}$ (Millenium Pharmaceuticals, Cambridge, MA, USA), the cleavage of the QFS was attributed to ACE2. The rate of substrate cleavage was expressed as picomoles of substrate cleaved per minute per milliliter of plasma.

\section{Cytokines assay}

The Predicta (Cambridge, MA) enzyme immunoassay kits were utilized to determine the levels of seminal IL-1 $\beta$, IL-6, IL-8, and TNF- $\alpha$. Levels of IFN- $\alpha$, IFN- $\gamma$, IL-10, and TGF- $\beta$ were determined using an ELISA method.

\section{ROS and SOD activity assay}

ROS levels were assessed by means of chemiluminescence assay using luminal (5-amino-2,3 dihydro-1,4 phtalazindione; Sigma Chemical Co. ) as the probe. An Autolamat LB 935 Luminometer (Berthold Technologies, Bad-wildbad, Germany) was used to ascertain ROS levels by measuring chemiluminescence activity in the integrated mode for $15 \mathrm{~min}$. Also, a commercially available colorimetric method (Randox Laboratories Ltd, Antrim, UK) was used to detect SOD activity based on the inhibition of 2-(4-iodophenyl)-3-(4-nitrophenol)5-phenyltetrazoliumchloride (I.N.T) reduction as a result of xanthine- and xanthine oxidase-generated superoxide radicals (Hajizadeh Maleki \& Tartibian 2020).

\section{Apoptotic variables assay}

Apo-Direct kit (Pharmingen, San Diego, CA, USA) was employed to detect sperm DNA fragmentation through a terminal deoxynucleotidyl transferase-mediated fluoresceindUTP nick end labeling (TUNEL) assay. In short, 1 to 2 million spermatozoa were washed in PBS, resuspended in $3.7 \%$ paraformaldehyde, adjusting the concentration to $1-2 \times 106$ $\mathrm{sperm} / \mathrm{mL}$, and kept on ice for 30 to $60 \mathrm{~min}$ at $4{ }^{\circ} \mathrm{C}$. Then, to remove the paraformaldehyde, spermatozoa were rewashed and after that resuspended in $70 \%$ ice-cold ethanol, and the specimens were placed at $-20^{\circ} \mathrm{C}$ until the run time. An internal set of tested negative or positive samples for DNA damage was also included with each run. The sperm pellets were resuspended in $50 \mu \mathrm{L}$ of the freshly prepared staining solution for $60 \mathrm{~min}$ at $37^{\circ} \mathrm{C}$ after a second wash in PBS to remove the ethanol. Based on the manufacturer's instructions, the staining solution contains TdT reaction buffer, fluorescein isothiocynate tagged dUTP nucleotides (FITC-dUTP), terminal deoxytransferase (TdT) enzyme, and distilled water. Further, to remove the unbound reaction solution, all specimens were washed in rinse buffer resuspended in a $0.5 \mathrm{~mL}$ of propidium iodide/RNase solution and then incubated in the dark at room temperature $30 \mathrm{~min}$. The flow cytometer FACScan (Becton Dickinson, San Jose, CA, USA) was used to analyze all fluorescence signals of labeled spermatozoa. At a flow rate of $<100$ cells/s, $~ 10,000$ spermatozoa were assessed for each assay. Supplied by an argon laser at $15 \mathrm{~mW}$, the excitation wavelength was $488 \mathrm{~nm}$. While the green fluorescence (480$530 \mathrm{~nm}$ ) was assessed in the FL-1 channel, the FL-2 channel was used to measure red fluorescence (580-630 nm). To exclude debris and aggregates, gating was done using $90^{\circ}$, and forward-angle light scatter. Using the flow cytometer software Flowlo version 6.2.4 (FlowJo, LLC, Ashland, OR, USA) and on a 1023-channel scale, the percentage of positive cells and the mean fluorescence, were calculated. On a 1023-channel scale, the flow cytometer software (Flow)o Mac version 8.2.4, FlowJo, LLC) was used to calculate the percentage of TUNELpositive spermatozoa (TUNEL ${ }^{+\Downarrow v e}$ ) (Hajizadeh Maleki \& Tartibian 2020). The ApoTarget Caspase-3/CPP32 Colorimetric protease assay was used to evaluate the proteolytic activity of caspase-3 in seminal plasma. Caspase-3 activity in seminal plasma was calculated according to the ratio of the optical density of caspase-3 to the protein concentration $(\mathrm{mg})$ of each 
cell lysate. Protein concentration was determined according to the previously developed technique (El-Melegy \& Ali 2011). Levels of caspase- 8 and caspase- 9 were determined using an ELISA method.

\section{Statistical analysis}

To detect significant $(P \leq 0.05)$ differences for study variables at four time periods among the groups, a repeated measure ANOVA was employed. Post-hoc Tukey analysis was used to identify differences between time points. Additionally, using an analysis of covariance (ANCOVA) adjusting for baseline measurements, differences among groups were detected. To assess the relationship between the quantitative variables studied, Pearson correlation coefficient and mixed linear regression model further were utilized. The statistical software program STATA 14 (StataCorp) was used for data analysis.

\section{Results \\ Description}

Among 84 patients who tested positive for COVID-19, $1.2 \%(1 / 84)$ were diagnosed as 'mild type', $27.4 \%(23 / 84)$ as 'moderate type', $32.1 \%$ (27/84) as 'severe type', and 39.3\% (33/84) as 'critical type'. The usages of corticosteroids and antiviral therapies were 44.0 (37/84) and 69.0\% (58/84), respectively. All the men were subjected to urological evaluation through a Urology expert and revealed normal outcomes on fertility assessment.

\section{Cytokines}

At the baseline and the subsequent follow-ups, the COVID-19 group had significantly higher levels of seminal IL-1 $\beta$, IL-6, IL-8, IL-10, TGF- $\beta$, INF- $\alpha$, and INF- $\gamma$ than the CON group $(P<0.05)$. At the baseline and 10 and 20 days, the COVID-19 patients had significantly higher levels of TNF- $\alpha$ than those in the CON group $(P<0.05)$, with no significant differences between the groups at 30 to 60 days $(P>0.05)$. Declined levels of IL- 6 and TNF- $\alpha$ were observed to the baseline at 20 days, while IL-1 $\beta$, IL-8, IL-10, TGF- $\beta$, INF- $\alpha$, and INF- $\gamma$ levels decreased from baseline at 30 days in the COVID-19 group $(P<0.05)$. No significant changes were observed in any of these cytokines within the CON group at any time points $(P>0.05)$ (Fig. 1$)$. The disease severity accompanied perturbations in the seminal cytokines.

\section{Peroxidative and antioxidative markers}

The COVID-19 group had significantly higher levels of seminal ROS and lower levels of SOD activity than the CON group at the baseline and at the subsequent follow-ups $(P<0.05)$. In the COVID-19 group, declining levels of ROS were observed compared to the baseline at 20 to 60 days $(P<0.05)$. No significant changes were observed in SOD activity across the seven-time points studied within the COVID-19 group $(P>0.05)$. Similarly, no significant differences were observed in any of these variables within the CON group at any time points $(P>0.05)$ (Fig. 2). Peroxidative and antioxidative markers were perturbated with the disease severity.

\section{Apoptotic variables}

Compared to the CON group, the COVID-19 patients had a significantly higher percentage of TUNEL ${ }^{+\otimes v e}$ as well as higher levels of caspase- 9 and caspase- 3 activity across the seven-time points studied $(P<0.05)$. Compared with baseline, the percentage of TUNEL ${ }^{+\otimes v e}$, caspase- 9 , and caspase- 3 activity tended to decline in the COVID-19 group at subsequent follow-ups, with significant improvements than baseline at 30 to 60 days $(P<0.05)$. At the baseline and 10 and 20 days, the COVID-19 patients had significantly higher levels of caspase-8 than the CON group $(P<0.05)$, with no significant differences between the groups at 30 to 60 days $(P>0.05)$. Declined levels of caspase- 8 were observed to the baseline at 20 to 60 days $(P<0.05)$. No significant changes were noticed in any of these variables at any time points within the CON group $(P>0.05)$ (Fig. 3; Supplementary figure 1, see section on supplementary materials given at the end of this article). The magnitude of the perturbations in the apoptotic variables was related to the disease severity.

\section{ACE2 activity}

The COVID-19 group had a significantly higher ACE2 activity across the seven-time points studied than the CON group $(P<0.05)$. Compared with baseline, ACE2 activity decreased in the COVID-19 group at subsequent follow-ups, with a significant decline than baseline at 30 to 60 days $(P<0.05)$. No substantial changes in ACE2 activity were observed within the CON group at any time points $(P>0.05)$ (Fig. 4$)$. The magnitude of the perturbations in the ACE2 activity was related to the disease severity.

\section{Semen quality parameters}

At the baseline and the subsequent follow-ups, the COVID-19 group had significantly lower levels of semen volume, progressive motility, sperm morphology, sperm concentration, and the number of spermatozoa compared to the CON group $(P<0.05)$. The enhanced number of spermatozoa was observed compared to the baseline at 10 to 60 days in the COVID-19 group, while progressive motility increased from baseline at 30 to 60 

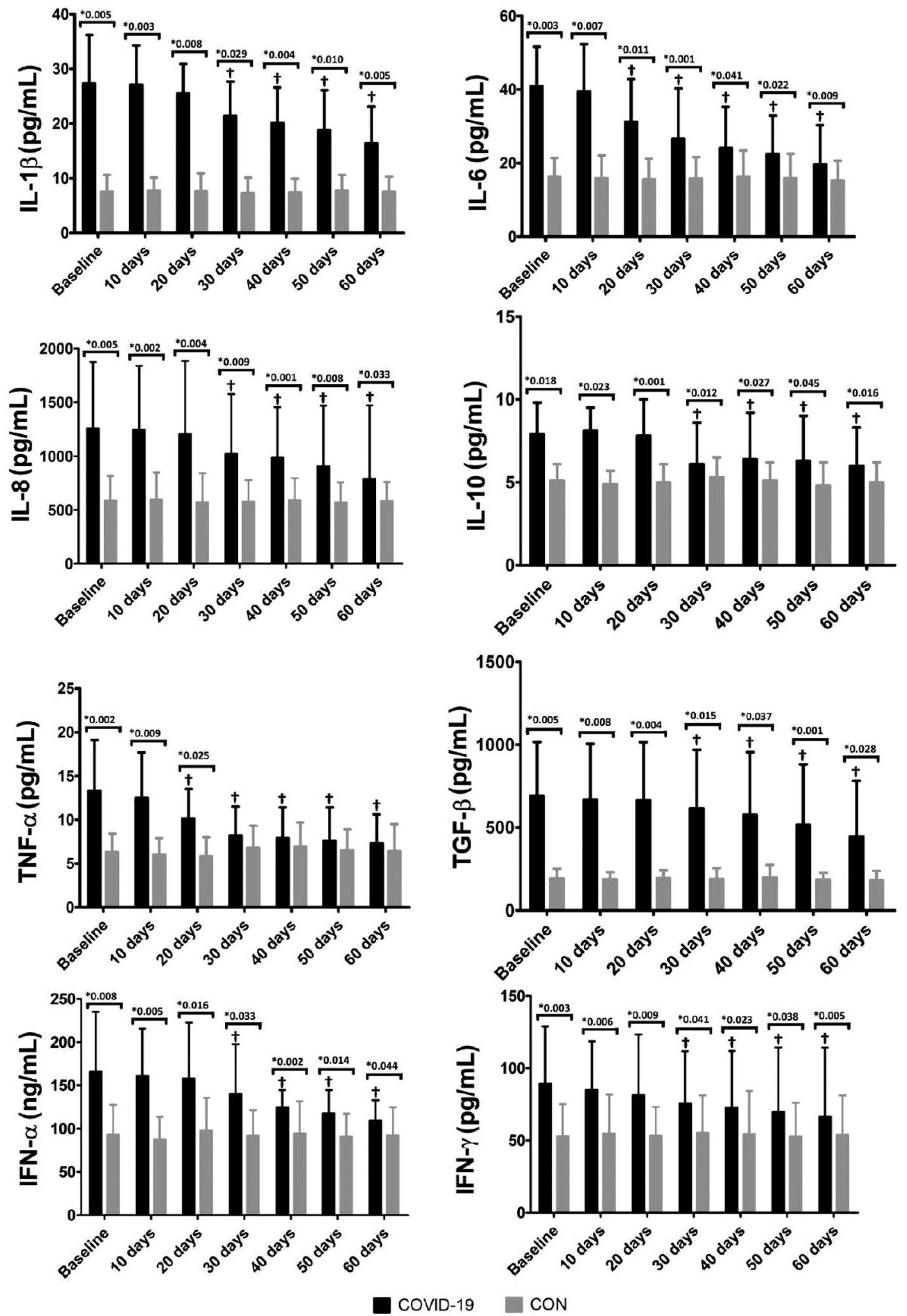

Figure 1 Changes in the seminal markers of inflammation in the COVID-19 and control (CON) groups during the study. The values are presented as the mean \pm S.D. ${ }^{\dagger} P<0.05$, significantly different from baseline values. ${ }^{*} P<0.05$, significant difference between the groups. 

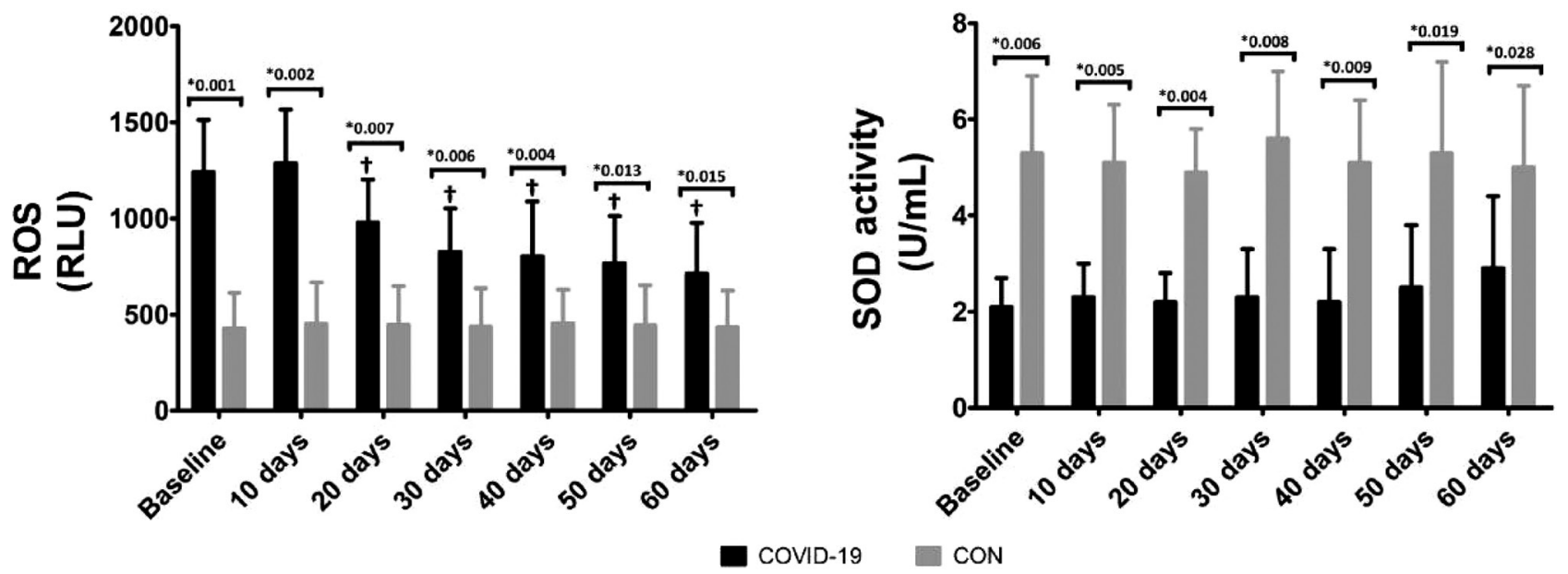

Figure 2 Changes in the seminal markers of oxidative stress in the COVID-19 and control (CON) groups during the study. The values are presented as the mean \pm S.D. ${ }^{\dagger} P<0.05$, significantly different from baseline values. ${ }^{*} P<0.05$, significant difference between the groups.
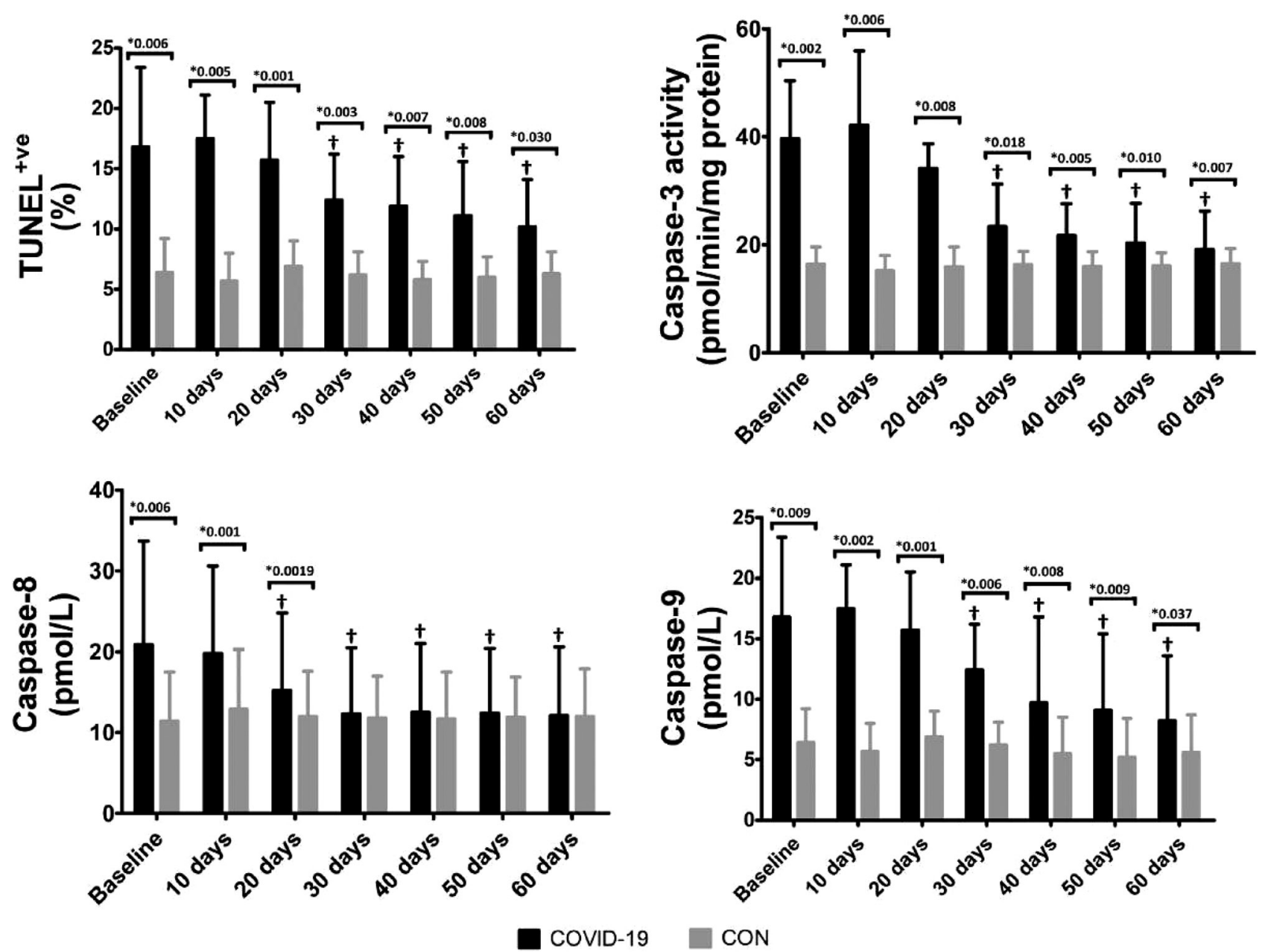

Figure 3 Changes in the seminal apoptotic variables in the COVID-19 and control (CON) groups during the study. The values are presented as the mean \pm S.D. ${ }^{\dagger} P<0.05$, significantly different from baseline values. ${ }^{*} P<0.05$, significant difference between the groups. 
days $(P<0.05)$. Across the seven-time points studied, no significant changes were seen in semen volume, sperm morphology, and sperm concentration within the COVID-19 group $(P>0.05)$. Similarly, no significant differences were observed in semen quality parameters within the CON group at any time points $(P>0.05)$ (Fig. 4; Supplementary Figure 2). The disease severity accompanied perturbated semen quality parameters.
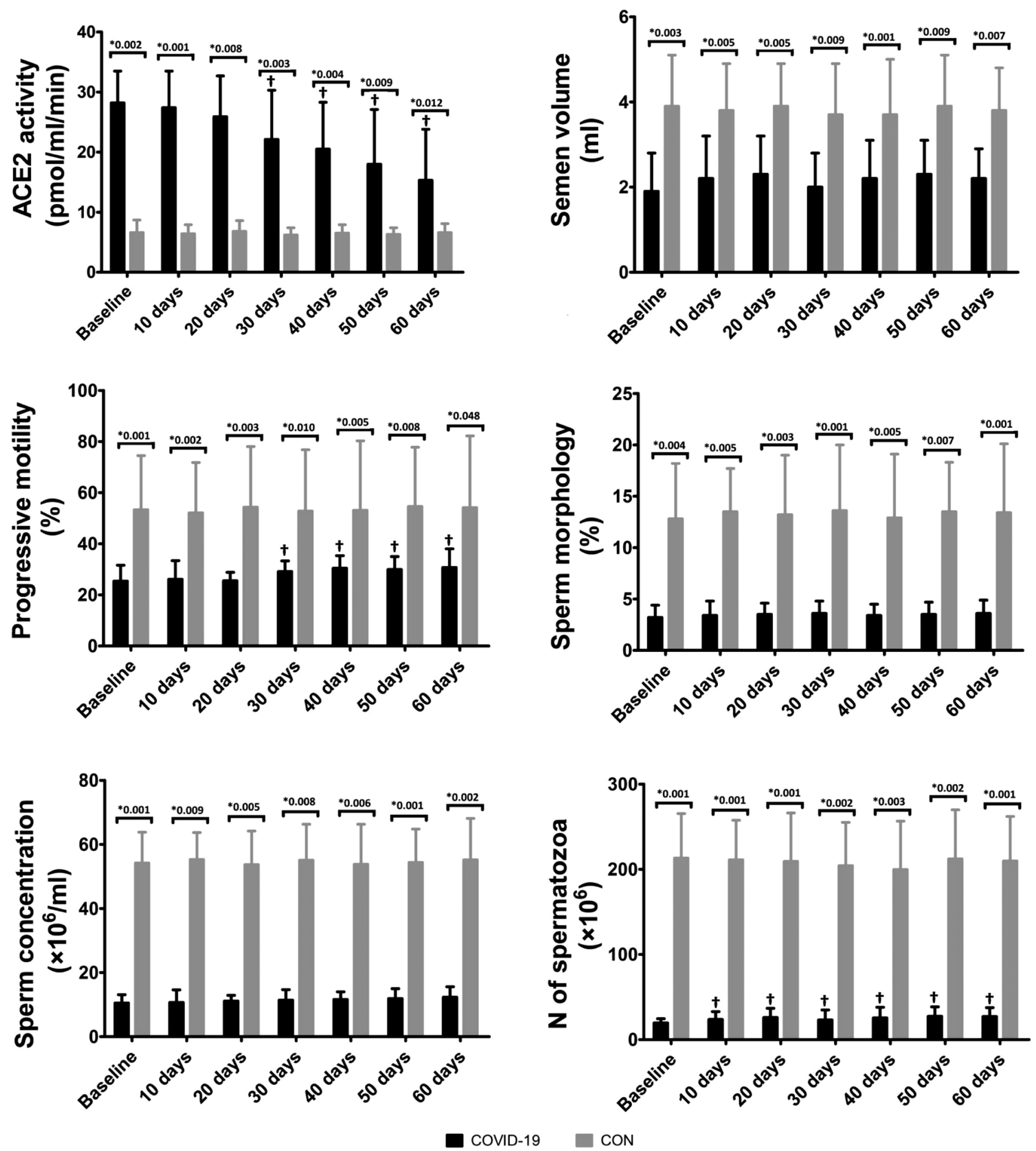

Figure 4 Changes in the seminal ACE2 activity and semen quality parameters in the COVID-19 and control (CON) groups during the study. The values are presented as the mean \pm S.D. ${ }^{\dagger} P<0.05$, significantly different from baseline values. ${ }^{*} P<0.05$, significant difference between the groups. 


\section{Correlations}

A significant negative correlation was observed between semen quality parameters with ACE2 activity, apoptotic variables, pro-and anti-inflammatory cytokines, and ROS in both groups. For each unit decrease in these variables, there were increased units in semen quality parameters. Likewise, significant positive correlations were observed between semen quality parameters with SOD. For each unit increase in SOD, there were increased units in semen quality parameters as well. Similarly, significant positive correlations were observed between ACE2 activity and apoptotic variables with pro-and anti-inflammatory cytokines and ROS. For each unit increase in ACE2 activity and apoptotic variables, there were increased units in these variables. Finally, significant negative correlations were observed between ACE2 activity and apoptotic variables with SOD. For each unit increase in SOD, there were increased units in these variables (Table 2).

\section{Discussion}

Our data demonstrate that COVID-19 infection causes significant impairments of male reproductive function. In detail, compared to the age-matched healthy controls, malepatients recovering from COVID-19 had significantly higher levels of seminal ACE2 enzymatic activity, pro-and anti-inflammatory cytokines, and apoptotic variables, as well as lower levels of SOD activity at the baseline. These perturbations persisted over time and were associated with significant impairments in semen quality parameters. This report provides direct evidence to date that COVID-19 infection impairs semen quality and male reproductive potential. The findings advance our understanding of the disease and are worthy of greater clinical attention and future systematic research.

Several independent research groups identified that every tissue in the human body distributing and expressing the ACE2 receptor might show the potential infection routes of COVID-19 and could be affected and damaged by the infection (Simoni \& Hofmann 2020, $\mathrm{Xu}$ et al. 2020). Our results revealed that the COVID-19 could significantly activate ACE2 in the seminal plasma that remains substantially higher during the subsequent measurements. The findings are consistent with the earlier reports (Köhn et al. 1995, Fan et al. 2020, Wang \& Xu 2020), where they discovered that ACE2 is highly expressed in the human testicular spermatogonia, Leydig and Sertoli cells, as well as in seminal fluid (Köhn et al. 1995). From these results, it is clear that the male reproductive system is a high-risk organ susceptible to the COVID-19 infection and should also be regarded as a potential target for the disease.

Our study of cytokine profiles of the seminal plasma revealed significantly higher levels of IL-1 $\beta$, IL-6, IL-8, TGF- $\beta$, TNF- $\alpha$, IFN- $\alpha$, and IFN- $\gamma$ in COVID-19 patients compared to the healthy CON group at the baseline. These primary findings tie well with earlier studies wherein the COVID-19 infection perturbates immunological characteristics and increases the systemic level of inflammatory mediators (Huang et al. 2020, Liu et al. 2020a). The excessive and dysregulated production of early response pro-inflammatory cytokines has been accepted as the disease's pathophysiologic hallmark. It brings about what has been characterized as a cytokine storm and explains the elevation of pro-inflammatory effector cytokines such as IL-1 $\beta$, IL-6, IL-8, and TNF- $\alpha$ in COVID-19 patients (Huang et al. 2020, Jose \& Manuel 2020). It has been reported that, in severe COVID-19 patients, the kinetic alterations of $\mathrm{T}$ cell counts were negatively linked with the kinetic variations of cytokine levels in the peripheral blood. Thus, the aggravated inflammatory responses during COVID-19 infection might be due to T cells' loss, as they play an essential role in dampening overactive innate immune responses during viral infections (Liu et al. 2020a). Produced by various cell types, IFN- $\alpha$ and IFN- $\gamma$ are involved in antiviral immune responses in a wide-ranging RNA and DNA viruses and carry out numerous functions during viral infections, including hampering replication and spread of the viral infection, as well as provoking cellular cytotoxicity and apoptosis in an organism (Samuel 2001). Therefore, increased interferons in COVID-19 patients highlight the importance of these mediators in viral infections, suggesting interferon-mediated protection against the viral infection in COVID-19 patients. Acting as a pro-viral factor, it has been shown that epithelialderived TGF- $\beta$ suppresses immune responses during early influenza A infection (Denney et al. 2018). Therefore, in the present study, it seems that increased levels of TGF- $\beta$ are a compensatory anti-inflammatory response to combat the excessive and dysregulated production of cytokine following COVID-19 infection. Regarding anti-inflammatory cytokine IL-10, our findings are in line with other studies that have shown a similar increase in IL-10 levels following COVID-19 infection (Huang et al. 2020). Thus, it seems the levels of IL-10 observed to be enhanced were stimulated possibly to deactivate the production of pro-inflammatory cytokines and protect reproductive cells against virally driven hyperinflammation. It is interesting to note that these perturbations were unabated during the 60 days of follow-up, suggesting that more extended periods are needed to recover the human male reproductive system's immune responses after the COVID-19 infection. The exact mechanisms of how COVID-19 infection changes seminal inflammatory mediators are not clear yet. Still, it seems that the perturbated immune factors may reflect the general immune response, like in serum.

Our study of oxidative and antioxidative parameters of the seminal plasma revealed that COVID-19 infection significantly impaired antioxidant defense machinery, as indicated by decreased levels of SOD activity, while 


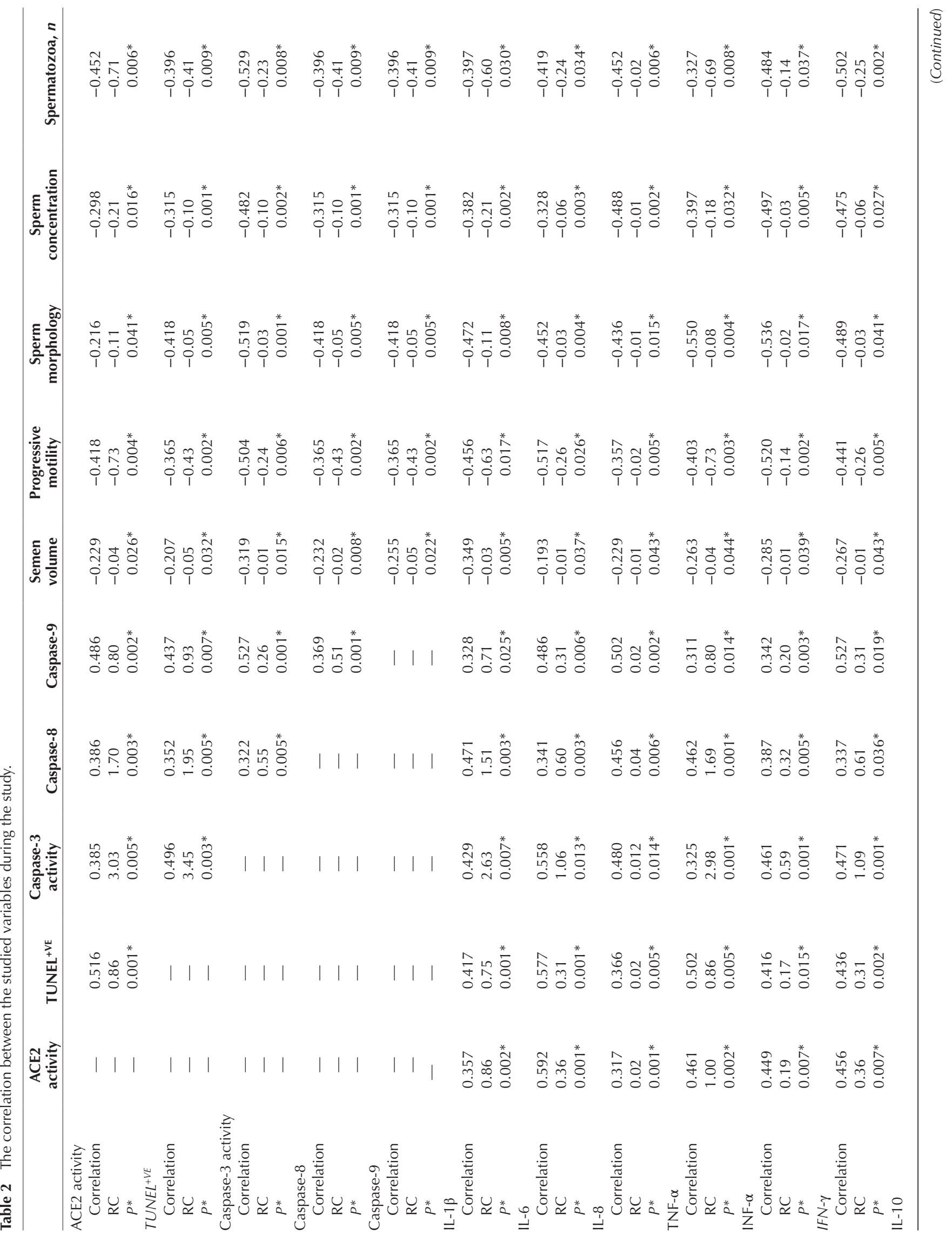




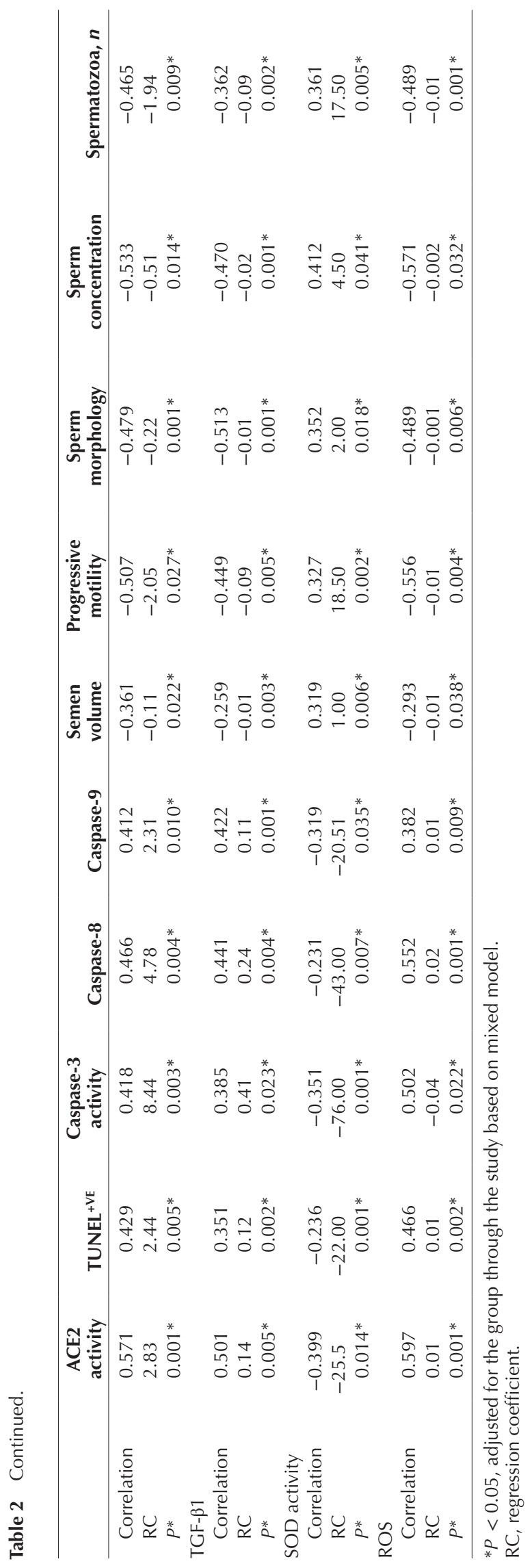

augmented oxidative stress responses, as demonstrated by an enhanced ROS production, were discovered. Clinical data regarding markers of oxidative stress and antioxidants in COVID-19 infection are limited. However, several independent lines of preclinical evidence suggest that the magnitude and the severity of SARS-CoV infection involvement are associated with the activation of oxidative stress mechanism that is connected with innate immunity and can induce an exacerbated pro-inflammatory host response (Smith et al. 2012). In the current study, it needs to be noted that the perturbations of seminal oxidative status persisted during the study and were accompanied by changes in pro-and anti-inflammatory mediators. Therefore, perturbation of seminal antioxidant defense machinery in the present study is likely related to the excessive and dysregulated activation of mediators of inflammation following COVID-19 infection.

To investigate whether COVID-19 infection caused cell death in sperm cells, we evaluated TUNEL activity in ejaculated human spermatozoa in male patients recovering from COVID-19. Remarkably, our current study demonstrated that the percentages of TUNEL+\&ve significantly increased in the seminal plasma of COVID19 infected patients early upon infection and remained substantially high over the subsequent follow-ups, suggesting that COVID-19 infection induces considerable cytopathological alterations, DNA damage, and apoptosis in the sperm cells. Also, to realize whether the COVID19 infection induces apoptosis in sperm cells, we assessed the activity of seminal caspase- 3 and seminal levels of caspase-8 and caspase-9, which represented the involvement of the extrinsic and intrinsic apoptosis pathway, respectively. Our results revealed that caspase-3 was substantially activated in the seminal plasma of COVID-19 infected patients early upon infection and stayed highly activated over time. Similar patterns were detected simultaneously in the caspases-8 and 9 levels, suggesting that the human spermatozoa were highly vulnerable to COVID-19 infection-induced apoptosis. Together, we demonstrated in the present study that COVID-19 infection-induced apoptosis in sperm cells was associated with the activation of caspases-3, 8, and 9 , signifying that both the execution phase of apoptosis as well as extrinsic and intrinsic apoptosis pathways were triggered in COVID-19 infection. Our findings, therefore, suggest that in the male patients recovering from COVID-19, the excessive and dysregulated production of inflammatory mediators along with a perturbation of seminal antioxidant defense system are necessary upstream signals for the apoptotic machinery, as higher levels of these mediators, have already been correlated with cytopathological alterations, DNA damage, and apoptosis in different cell types (Samuel 2001, Ramesh et al. 2009, Lambelet et al. 2018).

In the present study, semen quality parameters impaired early upon infection and remained significantly lower 
during the subsequent measurements in the COVID19 patients. These findings support the notion that the recovery of the male reproductive system from the COVID19 is incredibly low and undoubtedly needs more time for a complete revival. Another promising finding was that we observed semen quality parameters trending toward normal values as time went on; however, it seems that the infection caused a transient state of male subfertility like those with oligoasthenoteratozoospermia as they revealed abnormally lower levels of sperm concentration $(<15 \times 106 \mathrm{sperm} / \mathrm{mL})$, progressive sperm motility $(<32 \%$ motile), and sperm morphology $(<4 \%$ normal) during the study. In addition, semen quality parameters were positively correlated with SOD activity but negatively correlated with markers of inflammation and ROS. Therefore, from these results, we speculate that the observed impairments of semen quality parameters might be due to the COVID-19 infection-induced perturbations in the seminal inflammatory and redox responses.

It also has been reported that the patients' treatment with steroids, antiviral, and antiretroviral therapies able to cause testicular damage may have additional harmful consequences on semen quality parameters and function (Gür et al. 2005, Lorusso et al. 2010). Therefore, according to what has been reported above, concomitant treatments depict another possible mechanism to explain the detrimental effects of COVID-19 infection on the male reproductive system. Additionally, a febrile episode has been associated with adverse changes in semen quality parameters and sperm DNA integrity, which might be linked to future infertility (Sergerie et al. 2007). Consistent with this, as a febrile illness, high fever caused by COVID-19 infection might be responsible, at least in part, for the perturbated semen quality in this cohort of COVID-19 patients. Finally, it is important to highlight that these groups differed substantially in body weight, body fat percent, and BMl; therefore, it is possible that the reported effects were body composition related (Hajizadeh Maleki et al. 2019).

In the present work, we could not include other relevant parameters to this context like the male accessory gland (epididymis, prostate, and seminal vesicles) and urethral that are prone to viral infections and play a role in the male reproductive function and fertility. Therefore, with the current understanding of the disease, it is almost unclear whether these findings are attributed to the male reproductive tract-specific infection or merely the result of a general response to virus infection. However, the infection-induced activation of seminal ACE2 on the one hand and the potential role of ACE2 mRNA expression in the COVID-19 replication and pathogenesis, on the other hand, might, at least in part, show male tract-specific effects. Yet, a conservative interpretation of the findings is required because this protein has been predicted to be coexpressed only in a very small percentage in testicular cells, and the observed results in this study may be due to the COVID-19 disease. This is an interesting topic for future work, and further research is needed to validate and confirm if these are male reproductive tract-specific observations or merely the result of a general response to virus infection.

One concern about the findings of the present work was that we could not carry out the analysis based on the severity of disease for the mild type, as the number of patients in this category was inadequate; however, comparison among the moderate, severe, and critical types revealed that the magnitude of the perturbations in the studied variables was related to the disease severity. This is an important finding and sheds light on our understanding of the disease. Further, we did not measure hormonal levels in this study; however, a dramatic perturbation in testosterone ratio to luteinizing hormone, key players involved in male reproductive health, has previously been reported in the COVID19-infected male patients (Ma et al. 2020). Yet, despite these limitations, before this study, the association, and the infection-induced-longitudinal changes in the male reproductive function in COVID-19 patients were not clear. Importantly, this research adds weight to the prior findings and goes beyond our earlier understandings and shows that COVID-19 infection is significantly associated with impairments in the male reproductive function. Evidently, there is a crucial demand for further studies in bigger cohorts with prolonged follow-up periods to further investigate the consequences of COVID-19 infection on the male reproductive function.

In conclusion, in male patients recovering from COVID-19, we realized that the infection induces significant impairments in the male reproductive function, marked with an overproduction of the seminal markers of inflammation and oxidative stress as well as the activation of apoptotic variables. These changes were unabated during the subsequent measurements and were reversely correlated with semen quality parameters. We have also demonstrated that the COVID19 can activate ACE2 in the seminal plasma, suggesting that the semen of the patients should be considered as a vulnerable route to COVID-19 infection, and the reproductive functions of male recovering from the disease should be followed and evaluated since they may develop a transient state of male subfertility like those with oligoasthenoteratozoospermia.

\section{Supplementary materials}

This is linked to the online version of the paper at https://doi. org/10.1530/REP-20-0382.

\section{Declaration of interest}

The authors declare that there is no conflict of interest that could be perceived as prejudicing the impartiality of the research reported. 


\section{Funding}

The funder of the study had no role in study design, data collection, data analysis, data interpretation, or writing of the report. The corresponding authors had full access to all the data in the study and had final responsibility for the decision to submit for publication.

\section{Author contribution statement}

B H M and B T conceived and designed research; B H M analyzed data; B H M drafted manuscript; B H M and B T edited and revised manuscript; B H M and B T approved final version of manuscript.

\section{Acknowledgements}

The authors are grateful to the subjects' participation and dedication, without which the study could not have been carried out. The financial support of the Allameh Tabataba' $i$ University, Tehran, Iran is also gratefully acknowledged.

\section{References}

Chai X, Hu L, Zhang Y, Han W, Lu Z, Ke A, Zhou J, Shi G, Fang N \& Fan J 2020 Specific ACE2 expression in cholangiocytes may cause liver damage after 2019-nCoV infection. bioRxiv. (https://doi. org/10.1101/2020.02.03.931766)

Chan JF, Yuan S, Kok KH, To KK, Chu H, Yang J, Xing F, Liu J, Yip CC, Poon RW et al. 2020 A familial cluster of pneumonia associated with the 2019 novel coronavirus indicating person-to-person transmission: a study of a family cluster. Lancet 395 514-523. (https://doi.org/10.1016/ S0140-6736(20)30154-9)

Chen N, Zhou M, Dong X, Qu J, Gong F, Han Y, Qiu Y, Wang J, Liu Y, Wei $Y$ et al. 2020 Epidemiological and clinical characteristics of 99 cases of 2019 novel coronavirus pneumonia in Wuhan, China: a descriptive study. Lancet 395 507-513. (https://doi.org/10.1016/S01406736(20)30211-7)

Denney L, Branchett W, Gregory LG, Oliver RA \& Lloyd CM 2018 Epithelial-derived TGF- $\beta 1$ acts as a pro-viral factor in the lung during influenza A infection. Mucosal Immunology 11 523-535. (https://doi. org/10.1038/mi.2017.77)

Douglas GC, O'Bryan MK, Hedger MP, Lee DK, Yarski MA, Smith AI \& Lew RA 2004 The novel angiotensin-converting enzyme (ACE) homolog, ACE2, is selectively expressed by adult Leydig cells of the testis. Endocrinology 145 4703-4711. (https://doi.org/10.1210/en.2004-0443)

El-Melegy NT \& Ali ME 2011 Apoptotic markers in semen of infertile men: association with cigarette smoking. International Brazilian Journal of Urology 37 495-506. (https://doi.org/10.1590/s167755382011000400009)

Fan C, Li K, Ding Y, Lu WL \& Wang J 2020 ACE2 expression in kidney and testis may cause kidney and testis damage after 2019-nCoV infection. MedRxiv. (https://doi.org/10.1101/2020.02.12.20022418)

Gkogkou E, Barnasas G, Vougas K \& Trougakos IP 2020 Expression profiling meta-analysis of ACE2 and TMPRSS2, the putative anti-inflammatory receptor and priming protease of SARS-CoV-2 in human cells, and identification of putative modulators. Redox Biology 36 101615. (https:// doi.org/10.1016/j.redox.2020.101615)

Gür S, Bozkurt T \& Türk G 2005 Short term effects of dexamethasone on hyaluronidase activity and sperm characteristics in rams. Animal Reproduction Science 90 255-263. (https://doi.org/10.1016/j. anireprosci.2005.01.021)

Hajizadeh Maleki B \& Tartibian B 2020 High-intensity interval training modulates male factor infertility through anti-inflammatory and antioxidative mechanisms in infertile men: a randomized controlled trial. Cytokine 125 154861. (https://doi.org/10.1016/j.cyto.2019.154861)

Hajizadeh Maleki B, Tartibian B \& Chehrazi M 2019 Effects of aerobic, resistance, and combined exercise on markers of male reproduction in healthy human subjects: a randomized controlled trial. Journal of Strength and Conditioning Research 33 1130-1145. (https://doi. org/10.1519/JSC.0000000000002389)

Hoffmann $M$, Kleine-Weber $H$, Schroeder $S$, Krüger $N$, Herrler $T$, Erichsen S, Schiergens TS, Herrler G, Wu NH, Nitsche A et al. 2020 SARS-CoV-2 cell entry depends on ACE2 and TMPRSS2 and is blocked by a clinically proven protease inhibitor. Cell 181 271.e8-280.e8. (https://doi.org/10.1016/j.cell.2020.02.052)

Huang C, Wang Y, Li X, Ren L, Zhao J, Hu Y, Zhang L, Fan G, Xu J, Gu X et al. 2020 Clinical features of patients infected with 2019 novel coronavirus in Wuhan, China. Lancet 395 497-506. (https://doi.org/10.1016/S01406736(20)30183-5)

Jose RJ \& Manuel A 2020 COVID-19 cytokine storm: the interplay between inflammation and coagulation. Lancet: Respiratory Medicine 8 e46-e47. (https://doi.org/10.1016/S2213-2600(20)30216-2)

Köhn FM, Miska W \& Schill WB 1995 Release of angiotensin-converting enzyme (ACE) from human spermatozoa during capacitation and acrosome reaction. Journal of Andrology 16 259-265.

Lambelet M, Terra LF, Fukaya M, Meyerovich K, Labriola L, Cardozo AK \& Allagnat F 2018 Dysfunctional autophagy following exposure to proinflammatory cytokines contributes to pancreatic $\beta$-cell apoptosis. Cell Death and Disease 9 96. (https://doi.org/10.1038/s41419-017-0121-5)

Li H, Xiao X, Zhang J, Zafar MI, Wu C, Long Y, Lu W, Pan F, Meng T, Zhao K et al. 2020 Impaired spermatogenesis in COVID-19 patients. EClinicalmedicine 28 100604. (https://doi.org/10.1016/j. eclinm.2020.100604)

Liu J, Li S, Liu J, Liang B, Wang X, Wang H, Li W, Tong Q, Yi J, Zhao L et al. 2020a Longitudinal characteristics of lymphocyte responses and cytokine profiles in the peripheral blood of SARS-CoV-2 infected patients. EBiomedicine 55 102763. (https://doi.org/10.1016/j. ebiom.2020.102763)

Liu J, Liao X, Qian S, Yuan J, Wang F, Liu Y, Wang Z, Wang FS, Liu L \& Zhang Z 2020b Community transmission of severe acute respiratory syndrome coronavirus 2, Shenzhen, China, 2020. Emerging Infectious Diseases 26 1320-1323. (https://doi.org/10.3201/eid2606.200239)

Lorusso F, Palmisano M, Chironna M, Vacca M, Masciandaro P, Bassi E, Selvaggi Luigi L \& Depalo R 2010 Impact of chronic viral diseases on semen parameters. Andrologia 42 121-126. (https://doi.org/10.1111/ j.1439-0272.2009.00970.x)

Ma L, Xie W, Li D, Shi L, Mao Y, Xiong Y, Zhang Y \& Zhang M 2020 Effect of SARS-CoV-2 infection upon male gonadal function: a single centerbased study. MedRxiv. (https://doi.org/10.1101/2020.03.21.20037267)

Maya WDC \& Carvajal A 2020 SARS-CoV-2 and prostatitis: dangerous relationship for male sexual and reproductive health. Medical Hypotheses 144 109914. (https://doi.org/10.1016/j.mehy.2020.109914)

Mollica V, Rizzo A \& Massari F 2020 The pivotal role of TMPRSS2 in coronavirus disease 2019 and prostate cancer. Future Oncology 16 2029-2033. (https://doi.org/10.2217/fon-2020-0571)

Ramesh S, Wildey GM \& Howe PH 2009 Transforming growth factor $\beta$ (TGF $\beta$ )-induced apoptosis: the rise and fall of Bim. Cell Cycle 8 11-17. (https://doi.org/10.4161/cc.8.1.7291)

Ruan Y, Hu B, Liu Z, Liu K, Jiang H, Li H, Li R, Luan Y, Liu X \& Yu G 2020 No detection of SARS-CoV-2 from urine, expressed prostatic secretions, and semen in 74 recovered COVID-19 male patients: a perspective and urogenital evaluation. Andrology.

Samuel CE 2001 Antiviral actions of interferons. Clinical Microbiology Reviews 14 778-809. (https://doi.org/10.1128/CMR.14.4.778-809.2001)

Sergerie M, Mieusset R, Croute F, Daudin M \& Bujan L 2007 High risk of temporary alteration of semen parameters after recent acute febrile illness. Fertility and Sterility $\mathbf{8 8}$ 970.e971-970.e977. (https://doi. org/10.1016/j.fertnstert.2006.12.045)

Simoni M \& Hofmann MC 2020 The COVID-19 pandemics: shall we expect andrological consequences? A call for contributions to ANDROLOGY. Andrology 8 528-529. (https://doi.org/10.1111/andr.12804)

Smith JT, Willey NJ \& Hancock JT 2012 Low dose ionizing radiation produces too few reactive oxygen species to directly affect antioxidant concentrations in cells. Biology Letters 8 594-597. (https://doi. org/10.1098/rsbl.2012.0150) 
Wang Z \& Xu X 2020 scRNA-seq profiling of human testes reveals the presence of the ACE2 receptor, a target for SARS-CoV-2 infection in spermatogonia, Leydig and Sertoli cells. Cells 9 920. (https://doi. org/10.3390/cells9040920)

World Health Organization 2010 World Health Organization Laboratory Manual for the Examination and Processing of Human Semen, p. 287. Geneva, Switzerland: World Health Organization.

World Health Organization 2020 Clinical Management of Severe Acute Respiratory Infection When Novel Coronavirus ( $2019-n C o V) \bigotimes$ Infection is Suspected: Interim Guidance, 28 January 2020. World Health Organization.

Xu H, Zhong L, Deng J, Peng J, Dan H, Zeng X, Li T \& Chen Q 2020 High expression of ACE2 receptor of 2019-nCoV on the epithelial cells of oral mucosa. International Journal of Oral Science 12 8. (https://doi. org/10.1038/s41368-020-0074-x)

Zhang H, Kang Z, Gong H, Xu D, Wang J, Li Z, Cui X, Xiao J, Meng T \& Zhou W 2020 The digestive system is a potential route of 2019-nCov infection: a bioinformatics analysis based on single-cell transcriptomes. bioRxiv. (https://doi.org/10.1101/2020.01.30.927806)

Zhao Y, Zhao Z, Wang Y, Zhou Y, Ma Y \& Zuo W 2020 Single-cell RNA expression profiling of ACE2, the receptor of SARS-CoV-2. American Journal of Respiratory and Critical Care Medicine 202 756-759. (https:// doi.org/10.1164/rccm.202001-0179LE)
Zhou P, Yang XL, Wang XG, Hu B, Zhang L, Zhang W, Si HR, Zhu Y, Li B, Huang CL et al. 2020 A pneumonia outbreak associated with a new coronavirus of probable bat origin. Nature 579 270-273. (https://doi. org/10.1038/s41586-020-2012-7)

Zou X, Chen K, Zou J, Han P, Hao J \& Han Z 2020 Single-cell RNA-seq data analysis on the receptor ACE2 expression reveals the potential risk of different human organs vulnerable to 2019-nCoV infection. Frontiers of Medicine 14 185-192. (https://doi.org/10.1007/s11684-020-0754-0)

Zupin L, Pascolo L, Zito G, Ricci G \& Crovella S 2020 SARS-CoV-2 and the next generations: which impact on reproductive tissues? Journal of Assisted Reproduction and Genetics 37 2399-2403. (https://doi. org/10.1007/s10815-020-01917-0)

Received 8 July 2020

First decision 25 August 2020

Revised manuscript received 29 December 2020

Accepted 21 January 2021 